\title{
Cytomegalovirus Retinitis in an Immunocompromised Infant: A Case Report and Review of the Literature
}

\author{
A. Mota ${ }^{a} \quad$ J.Breda ${ }^{a} \quad$ R. Silva ${ }^{a, b} \quad$ A. Magalhães ${ }^{a} \quad$ F. Falcão-Reis ${ }^{a, b}$

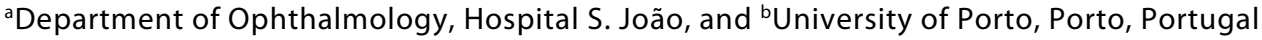

\section{Key Words}

Cytomegalovirus · Retinitis · Infant · Ganciclovir

\begin{abstract}
Purpose: To describe a case of bilateral cytomegalovirus retinitis (CMVR) in an immunocompromised infant.
\end{abstract}

Methods: A 4-month-old male infant with severe combined immunodeficiency syndrome was examined for the presence of CMVR. Ocular involvement was recorded and monitored by digital imaging.

Results: The child had bilateral CMVR, with a fine granular pattern, present both in the peripheral retina and posterior pole. There was no vitritis. The active areas of retinitis progressively resolved with intravenous ganciclovir treatment. At the 3-month follow-up examination, no recurrence was observed.

Conclusion: Ganciclovir treatment was effective in this case. The prognosis depends on rapid institution of effective antiviral therapy and on a patient's systemic immunocompetence.

\section{Introduction}

Cytomegalovirus (CMV) is the most frequent cause of congenital and perinatal viral infections in humans [1]. The incidence of CMV retinitis (CMVR) in children with systemic disease has been reported to be as high as $25 \%$ in infants with severely symptomatic congenital CMV and 1\% in asymptomatic congenitally infected infants [2]. CMVR is the most common opportunistic infection in the eyes of adults with HIV, and before combination therapy $20-40 \%$ of these patients were affected. In contrast, CMVR has been reported in only $5 \%$ of children with HIV [3]. Other immunosuppressive conditions associated with CMVR in children are severe combined immunodeficiency 
syndrome (SCID), acquired immunodeficiency syndrome after bone marrow or renal transplantation, and chemotherapy [4]. The occurrence of CMVR is less common in children than in adults. Only few cases have been reported in children. We describe a case of an immunocompromised infant with acquired systemic CMV infection who developed bilateral CMVR.

\section{Case Report}

A 4-month-old male infant with a recent diagnosis of SCID developed an acute CMV infection with interstitial pneumonitis and colitis. Initial PCR detection of CMV DNA in serum was positive, with 5,800 copies $/ \mathrm{ml}$. The patient was referred to our institute for the evaluation of possible CMVR. He was admitted to a pediatric intensive care unit, and treatment for all the systemic problems described was started, including intravenous immunoglobulin once a week along with intravenous ganciclovir (10 $\mathrm{mg} / \mathrm{kg} /$ day).

He was examined 1 week after the initiation of treatment. Fundus photographs were made with a RetCam 120 wide-field digital fundus camera (Massie Labs, Dublin, Calif., USA). Our patient had bilateral active CMVR with multiple granular whitish lesions, present in the peripheral retina and posterior pole (fig. 1). Progressive pigment deposition was found around the lesions from posterior to anterior, and evolved to chorioretinal scarring (fig. 2 , fig. 3). Reevaluation was done weekly until total resolution of the active retinal lesions could be observed. The patient was followed up for 3 months and no recurrence was found. Treatment with intravenous ganciclovir $(10 \mathrm{mg} / \mathrm{kg} / \mathrm{day})$ was continued during hospitalization (12 weeks). The child was then transferred to another hospital unit to undergo a bone marrow transplantation, which is the treatment of choice for SCID.

When he was discharged, the patient's IgG levels were $841 \mathrm{mg} / \mathrm{dl}$ and there were $900 \mathrm{copies} / \mathrm{ml}$ of CMV DNA. Ganciclovir dosage was not reduced due to still active systemic CMV infection.

\section{Discussion}

The disease patterns of CMVR are quite different in infants compared to adults. Baumal et al. [5], in a review of 9 children with CMVR, found that it was bilateral in $89 \%$ and involved the posterior pole in at least 1 eye of all 9 patients. Wren et al. [3] recently confirmed this pattern of presentation. In our case, there was bilateral involvement with lesions in the posterior pole. The fundal appearances may vary from subtle white flecks, giving a granular appearance, to more florid presentations with white infiltrates accompanied by hemorrhage [3]. Congenital infection is typically less hemorrhagic than perinatal infection acquired by immunocompromised children. However, our case had only multifocal granular lesions, without hemorrhage or vitritis. Factors that may contribute to the differences in disease expression are the state of the immune system, the type of exposure to the virus, and primary infection/reactivation of the latent virus.

Prompt treatment is probably the most important prognostic factor; however, the optimal therapy for CMVR in children has not yet been defined [1]. Ganciclovir has potential toxicity as it can cause neutropenia, inhibit spermatogenesis and induce possible carcinogenic effects in animals. Long-term safety data regarding its use in infants are not yet available [2]. Most clinical trials with ganciclovir have shown reduced hearing loss and regression of retinitis. The use of oral valganciclovir as an alternative therapy is currently under evaluation since its bioavailability equals that of intravenous ganciclovir and it does not have the associated comorbidities of prolonged hospital stay and intravenous administration $[6,7]$. Ohta et al. [8] showed the efficacy and safety of foscarnet for 
treating pediatric patients with CMVR. Current treatment for CMV infection is intravenous ganciclovir for 3-6 weeks; however, there are still doubts regarding which infants should be treated and for how long. In immunocompromised infants with symptomatic CMV infection, treatment should be initiated as soon as possible to prevent long-term systemic or visual morbidity.

In our case, treatment was based on a defective immune system, viremia and organ damage. CMVR was detected 1 week after starting treatment and, therefore, the lesions showed some initial scarring characteristics. We believe that prompt treatment was decisive to prevent progression to a more serious form of chorioretinitis.

\section{Disclosure Statement}

The authors have no sponsorship or funding arrangements relating to research and no conflicts of interest to declare.
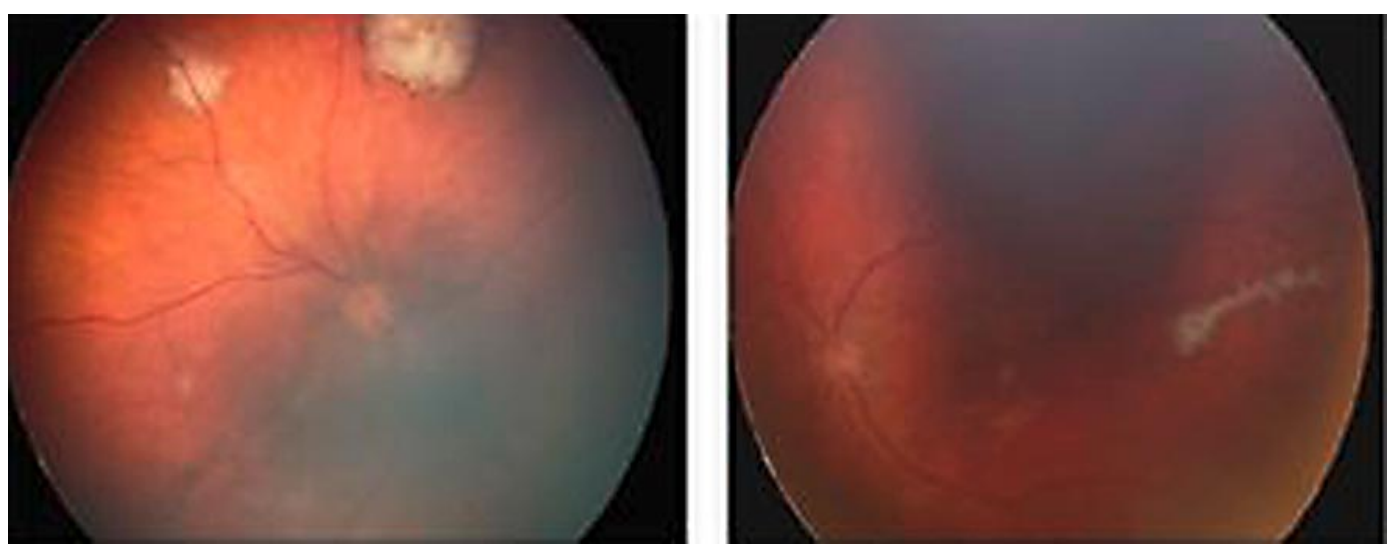

Fig. 1. Right fundus showing granular whitish lesions, two in the retinal periphery and one in the posterior pole. The largest lesion already shows some pigmentation in the periphery. Left fundus showing granular whitish lesions in the posterior pole. 

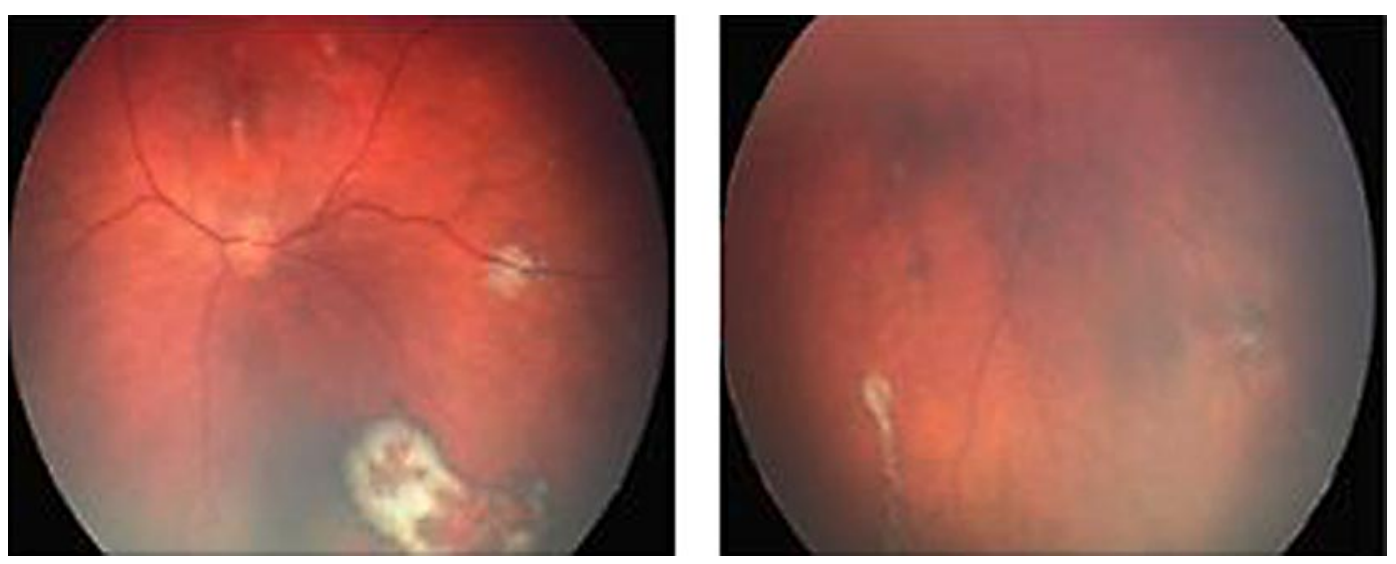

Fig. 2. Two weeks after initiating treatment, the right and left fundus showed progressive pigmentation of the lesions.
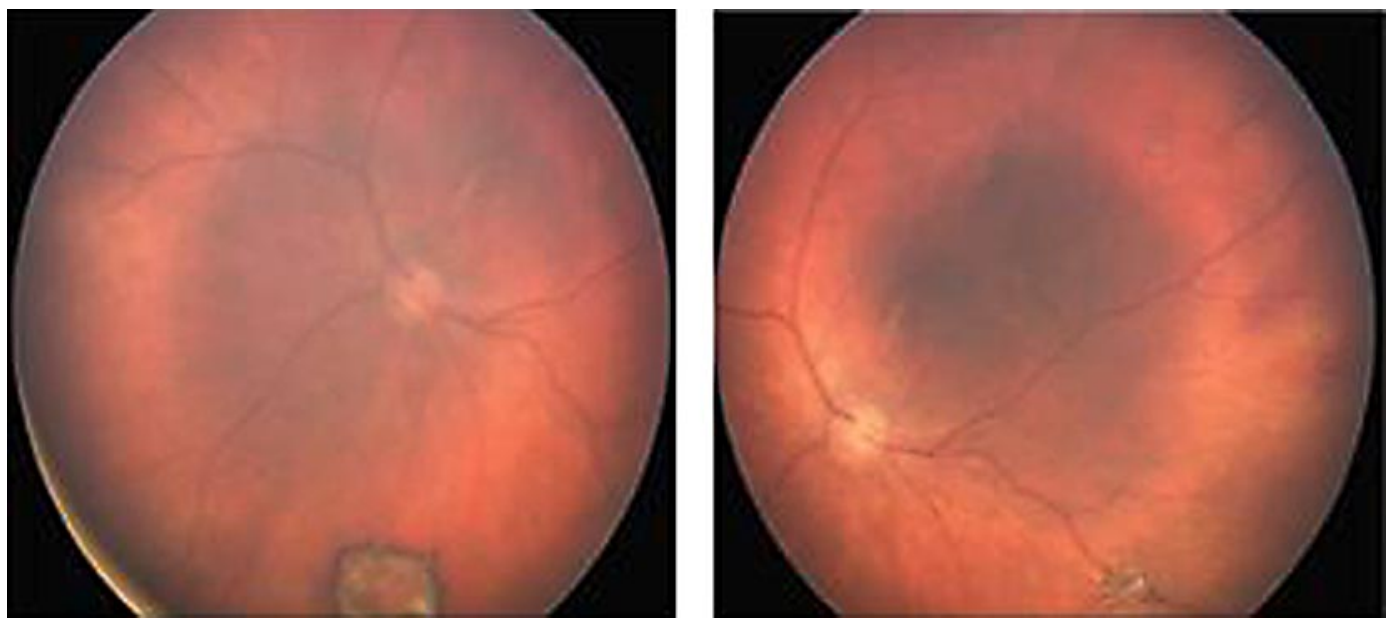

Fig. 3. One month after treatment, right and left fundus images showed total resolution of the active lesions, with the formation of chorioretinal scars. 


\section{References}

1 Barampouti F, Rajan M, Aclimandos W: Should active CMV retinitis in non-immunocompromised newborn babies be treated? Br J Ophthalmol 2002;86:248-249.

$\checkmark 2$ Coors LE, Spencer R: Delayed presentation of cytomegalovirus retinitis in an infant with severe congenital cytomegalovirus infection. Retina 2010;30:S59-S62.

-3 Wren SM, Fielder AR, Bethell D, Lyall EG, Tudor-Williams G, Cocker KD, Mitchell SM: Cytomegalovirus retinitis in infancy. Eye (Lond) 2004;18:389-392.

4 Baumal CR, Levin AV, Kavalec CC, Petric M, Khan H, Read SE: Screening for cytomegalovirus retinitis in children. Arch Pediatr Adolesc Med 1996;150:1186-1192.

5 Baumal CR, Levin AV, Read SE: Cytomegalovirus retinitis in immunosuppressed children. Am J Ophthalmol 1999;127:550-558.

6 Burri M, Wiltshire H, Kahlert C, Wouters G, Rudin C: Oral valganciclovir in children: single dose pharmacokinetics in a six-year-old girl. Pediatr Infect Dis J 2004;23:263-266.

-7 Meine Jansen CF, Toet MC, Rademaker CM, Ververs TF, Gerards LJ, van Loon AM: Treatment of symptomatic congenital cytomegalovirus infection with valganciclovir. J Perinat Med 2005;33:364-366.

-8 Ohta H, Matsuda Y, Tokimasa S, Sawada A, Kim JY, Sashihara J, Amo K, Miyagawa H, Tanaka-Taya K, Yamamoto S, Tano Y, Aono T, Yamanishi K, Okada S, Hara J: Foscarnet therapy for ganciclovir-resistant cytomegalovirus retinitis after stem cell transplantation: effective monitoring of CMV infection by quantitative analysis of CMV mRNA. Bone Marrow Transplant 2001;27:1141-1145. 